\title{
The Synthesis of Electric Drives Characteristics of the UAV of "Convertiplane-Tricopter" Type
}

\author{
O. V. Emelyanova ${ }^{1,{ }^{*}}$, G. K. Kazaryan ${ }^{1}, A . S$. Martinez Leon ${ }^{1}$, and S. F. Jatsun ${ }^{1}$
}

${ }^{1}$ Southwest State University, 305040 Kursk, Russia

\begin{abstract}
The paper is devoted to the review of controlled motion of the unmanned aerial vehicle (UAV) with vertical takeoff and landing, the convertiplane type, with three tilt rotors. The UAV is equipped with a swept wing, the calculation of aerodynamic parameters of which has been carried out in the software program XFLR5, taking into consideration the weight and size requirements. There have been determined the lift and resistance coefficients of the UAV depending on the angle of attack on the basis of the vortex method. There has been done the analysis of the influence of wing profile on the calculated aerodynamic characteristics. There also has been realized the synthesis of the traction energy-efficient options of the drive propellers that reduce power consumption, increasing the accuracy of the automatic control system.
\end{abstract}

\section{Introduction}

Currently, the UAVs of various structural and constructive models, performing vertical takeoff and landing, are actively used in many spheres of human activity. It is connected with the fast growing technology and engineering solutions. As the result, such devices have become compact and maneuverable, simple in production and controlling, with a reliable and lightweight construction that has been achieved through the use of modern composite materials, providing a low weight of these devices with a significant payload mass $[1-4,5,11]$.

Convertiplane can be considered as one of the most promising types of UAVs, because it combines helicopter features - a vertical takeoff (landing), and aircraft features - a flight in a horizontal plane if there is a wing, by changing the traction vector of propellers [5$8,13-15]$. The advantage of such device is the energy consumption reduction of the on-board system that can be achieved by turning the traction forces of the propellers in the direction of movement, and using the flying wing, as the significant part of the aerodynamic force is formed upon it. The convertiplane flight can be divided into several stages: the climb and decrease on the vertical trajectory, and the flight in the horizontal plane. The modes of vertical takeoff or landing are more complex than a horizontal flight, as there is an additional resistance of the fuselage to the wing. In addition, the fuselage of the convertiplane has a bad streamlining during the vertical rise. Therefore, in these modes, the traction of the lifting propellers increases substantially. [1-4].

The horizontal flight of a convertiplane requires less traction, because in this case the fuselage and the wings are streamlined by air and their frontal resistance decreases. The resistance of the lifting propellers also reduces as a result of their transition to a smaller angles of attack or the turning in the direction of movement. At any speed of the convertiplane horizontal movement the lift of the wing allows to minimize the height loss at the moment of the propellers turning, providing the necessary stability of the flying vehicle. In this case an important role plays the calculation of the aerodynamic parameters of the wing, which can significantly increase the carrying capacity of the convertiplane during a horizontal flight.

\section{Description and principle of movement of the convertiplane}

Let's consider the motion of a convertiplane in a fixed cartesian coordinate system $O X Y Z$, where $C X_{1} Y_{1} Z_{1}$, $A_{i} x_{i} y_{i} z_{i}(i=1,2,3)$ are movable coordinate systems passing through the mass center of the device body $C$ and the mass center of the $i$-rotor, respectively (Fig. 1). The space orientation is set by the plane yaw $\psi$, pitch $\theta$ and roll $\varphi$ angles.

The device consists of three pairs of controlled propellers 1-3, on the basis of the brushless electric drive with variable traction vectors and the fuselage 4 , on which there also fixed a power supply, a control board and a signal receiver that are electrically connected with the rotary drive of the propellers.

The declination of the rotary drive traction vectors 1 , 2 is carried out in the plane $A_{i} X^{\prime}{ }_{1} Z_{1}^{\prime}\left(\| C X_{1} Z_{1}\right)$ at an angle $\beta$, which may vary from 0 to $90^{\circ}$, and the drive $3-$ in the plane $A_{3} Y^{\prime}{ }_{1} Z^{\prime}{ }_{1} \quad\left(\| C Y_{1} Z_{1}\right)$ at an angle $\pm 45^{0}$. 
The presence of the traction horizontal component of the tail drive will allow providing the rotation of the convertiplane around the vertical axis $C Z_{1}$ on the yaw angle $\psi$. The torque compensation takes place due to the oppositely installed propellers, turning in opposite directions $[8,9]$.

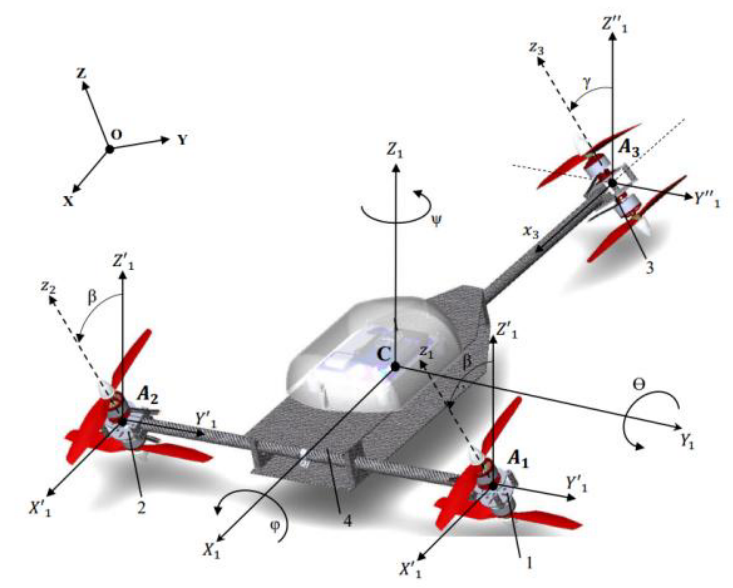

Fig. 1. The general view of a convertiplane-tricopter power frame.

\section{Calculation of the convertiplane aerodynamic parameters}

When calculating the basic convertiplane performance characteristics, as well as its stability and controllability, it is necessary to know the forces acting on the device during the flight. Conditionally, they can be divided into the following forces (Fig.2) [5-7]:

1) in absolute (Cartesian) coordinate system $O X Y Z$ :

- the gravity $\bar{G}$ of the convertiplane applied in the mass center $C$;

2) in a moving coordinate system $C X_{I} Y_{1} Z_{1}$ :

- the forces, generated by the propellers $\bar{F}_{1}-\bar{F}_{3}$, applied in the mass center of the rotors $A_{1}-A_{3}$;

- the aerodynamic forces, acting on the wing surface, called the force of frontal resistance $\bar{Q}_{i}$, directed to the opposite side of the axis $C X_{1}$, and the lift force $-\bar{P}_{i}$, directed along the axis $C Z_{1}$ (Fig. 2) and, similarly, the aerodynamic forces, acting on the fuselage: $\bar{Q}_{\Phi}$ and $\bar{P}_{\Phi}$.

The aerodynamic forces, acting on the wing surface, can be expressed via dimensionless coefficients of the frontal resistance $C D$ and the lift CL. Due to the smallness of the side force coefficient $\mathrm{CP}$, the aerodynamic side force $\bar{Q}$, won't be taken into account in this modelling [3].

Then

$$
\left[\begin{array}{l}
Q \\
P
\end{array}\right]=q_{\infty} S\left[\begin{array}{l}
C_{D} \\
C_{L}
\end{array}\right] .
$$

where $q_{\infty}=\rho v_{\infty}^{2} / 2$ the dynamic velocity pressure; $v_{\infty}-$ the air rate; $\mathrm{S}-$ the wing area.

The aerodynamic characteristics of the wing depend on the geometric parameters of the wing profile, the orientation of the wing in the airflow (angle of attack $\alpha$ ), similarity parameters, etc. Therefore, for their determination the results of analytical, numerical and experimental studies, founded in XFLR5 software product under specified geometric and aerodynamic characteristics of the airfoil will be used.

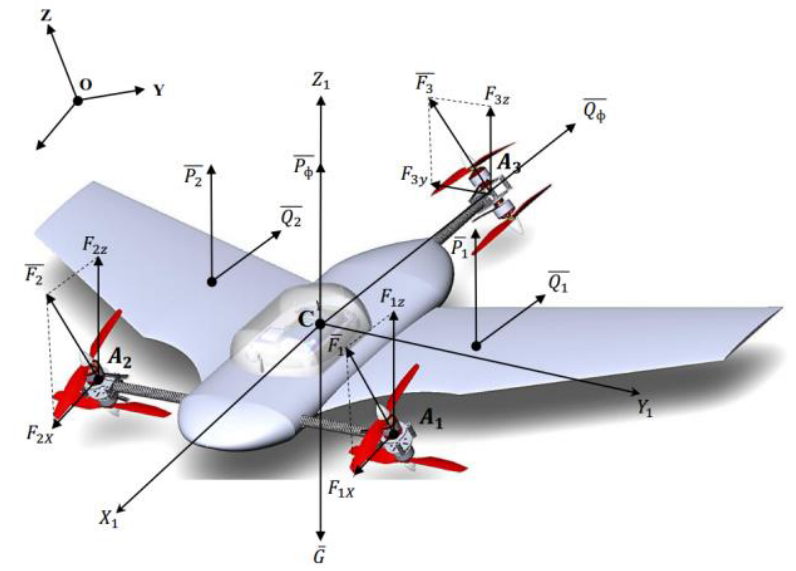

Fig. 2. The scheme of forces, acting on a convertiplane during the flight

The selection of the airfoil is one of the main quality indicators in the study of the UAV aerodynamic characteristics. For the flying vehicle under review the selection of the profiles has been made from the standard (normal) profile classes "FX" and "NACA", which, if necessary, can be modified at the stage of conceptual design (Fig. 3). This choice is determined by the fact, that the profiles of represented classes have low weight, acceptable thickness, the maximum time of floating in the air, the highest lifting capacity of all the standard series, the stability at low and high values of the number Re (laminar and turbulent flow) [13]. The profiles are asymmetric, because the pressure difference, occurring at the lower and upper edges, provides a greater value of the lift force than on the symmetrical ones.

Further, the effect of the selected wing profiles and the swept wing shape on the main vector of the aerodynamic forces will be considered. The choice of a swept wing shape is determined by the fact, that it has a high lift coefficient at small angles of attack $[1,3]$. This allows reducing its dimensions and weight. For modeling the shape, there have been used the following geometric parameters (Fig. 4): the root chord $\left(b_{0}\right)$, the mean chord $\left(b_{c p}\right)$, the tip chord $\left(b_{k}\right)$, the sweep angle $(\alpha)$ and the wing length ( $\left.l_{\text {wing }}\right)$.

Next, there will be determined the optimum ratio that provide the maximum value of the lift coefficient $C_{L}$ from the angle of attack $\alpha$ for different types of profiles. The modelling has been carried out with the following parameters: the air density $\rho=1,225 \mathrm{~kg} / \mathrm{m}^{3}$, the speed of the oncoming air flow $v=5 \mathrm{~m} / \mathrm{s}$. The modelling results are shown in Fig.5. 


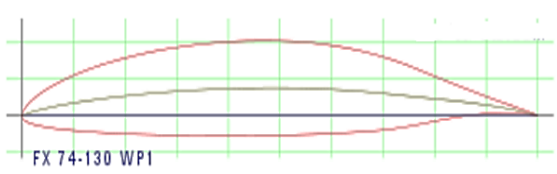

a

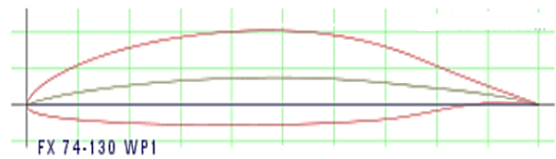

b

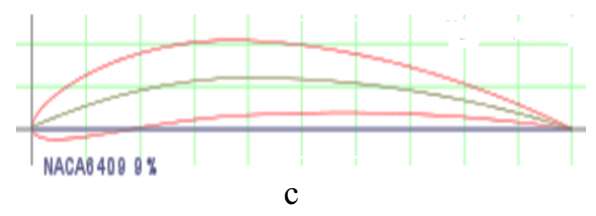

Fig. 3. The profiles under studi: $a-$ FX 74-130; b - FX74CL5-140; $c$ - NACA 6409 9\%

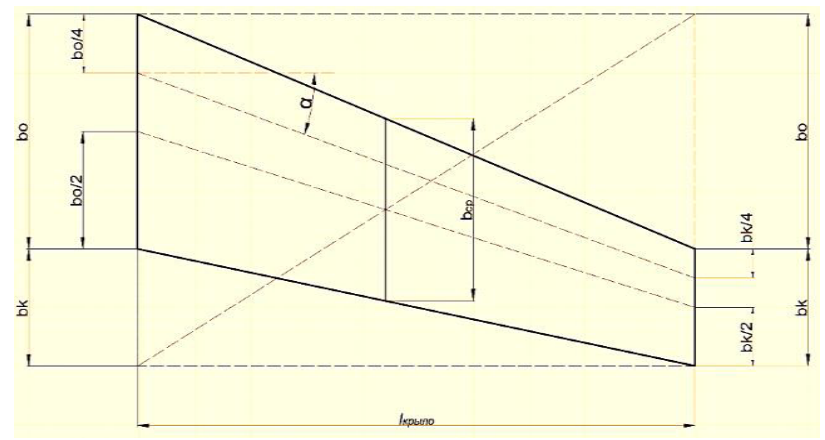

Fig. 4. The geometric parameters of a planned wing

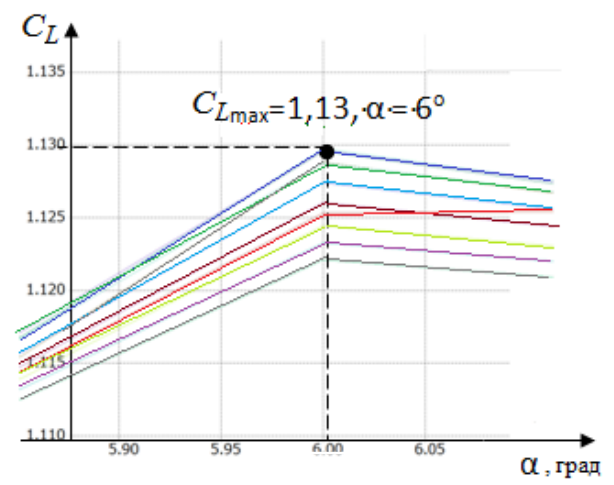

a

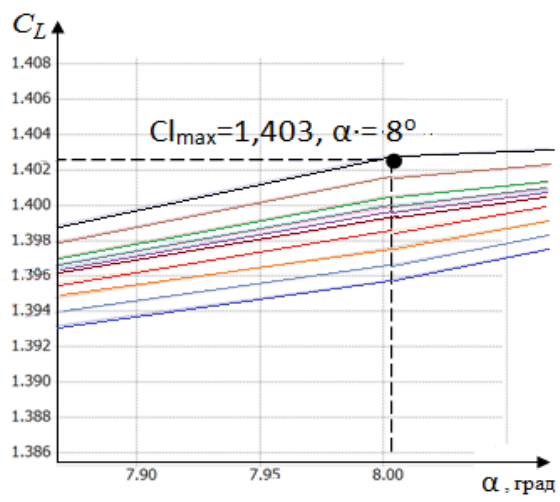

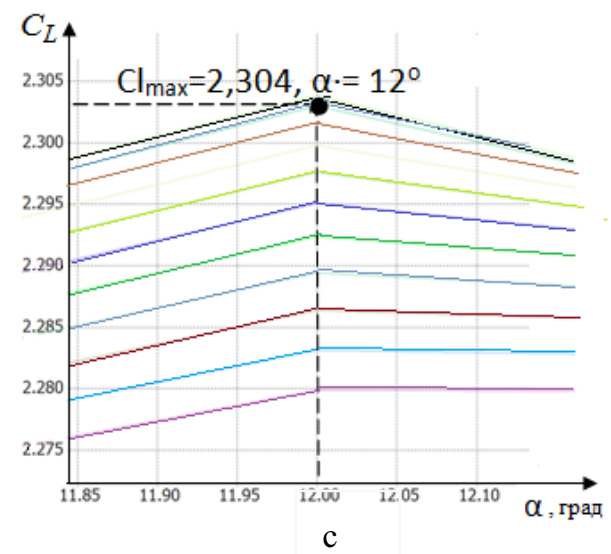

Fig. 5. The lift coefficient $C_{L}-$ angle of attack $\alpha$ diagram, using the profiles: $a-$ FX 74-130; $b$ - FX74-CL5-140; $c-$ NACA 6409

Similarly, there will be determined the optimum ratio, providing the maximum value of the resistance coefficient $\mathrm{CD}$ from the angle of attack $\alpha$ for different types of profiles.

Then, taking into consideration (1), there will be calculated the aerodynamic lift and frontal resistance forces for the swept wing shape and different profiles. The results of the study are presented in table 1 .

Table 1. The aerodynamic parameters of the wing

\begin{tabular}{|c|c|c|c|c|c|c|c|}
\hline Form & Profile & $\begin{array}{l}\alpha \\
{\left[{ }^{0}\right]}\end{array}$ & $\mathrm{Cl}$ & Cd & $\begin{array}{c}S \\
{\left[\mathrm{~m}^{\wedge} 2\right]}\end{array}$ & $\overline{\boldsymbol{P}},[\mathrm{H}]$ & $\overline{\mathbf{Q}},[\mathrm{H}]$ \\
\hline & $\begin{array}{c}\text { FX74- } \\
130\end{array}$ & 6 & 0,81 & 0,03 & \multirow{3}{*}{0,22} & 2,72 & 0,11 \\
\hline & $\begin{array}{c}\text { FX75- } \\
\text { CL5-140 }\end{array}$ & 8 & 1,44 & 0,10 & & 4,83 & 0,35 \\
\hline $\begin{array}{l}\text { Arrow- } \\
\text { shape }\end{array}$ & $\begin{array}{c}\text { NACA } \\
64099 \%\end{array}$ & 12 & 1,33 & 0,88 & & 4,48 & 2,96 \\
\hline
\end{tabular}

According to the modelling results there will be chosen the wing with the following characteristics: the profile - NACA $64099 \%$; the wingspan - $1 \mathrm{~m}$; the mass of the wing $-0,25 \mathrm{~kg}$.

The slow growth of the lift force, which depends on the angle of attack, provides stability to the turbulence of the air flow. The range of angles of attack, ensuring a stable flight, is $6-12 \mathrm{o}$. Large values can lead to the disruption of the airflow stream, the lift coefficient growth fall of the convertiplane $[1,3,14]$.

\section{The description and principle of movement of the convertiplane}

In this part there will be analyzed the electro-mechanical system of the tricopter propeller drives (Fig.6) [9]. The propellers are driven by electric motors that generate the moments $M_{E M i}$, while the moment of external loads $M_{H i}$, prevents the rotation of the propellers with angular velocities $\omega_{i}$. 
The restoring moment, acting on the frame from the electric drives of propellers, is determined by the value of the control voltages, coming from the automatic control system on the electric drives of the four propellers. And the level of voltages, generated by the power amplifier of the automatic control system, entirely depends on the type of controller.

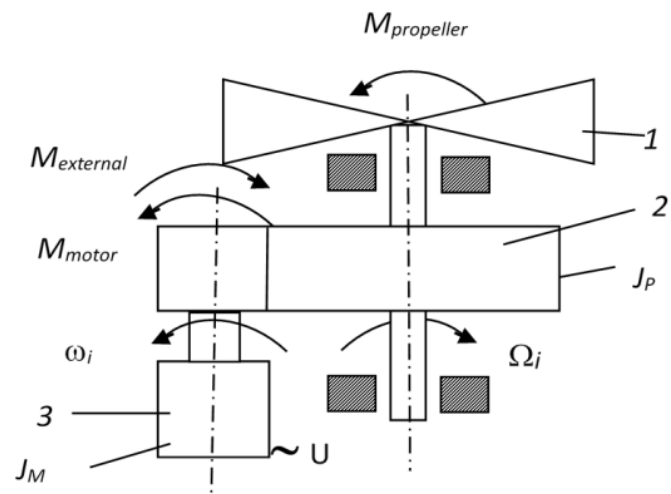

Fig. 6. The simplified scheme of the propeller electric drive: 1 - propeller; 2 - reducer; 3 - electric motor; U - control voltage; $M_{\text {motor }}$ - the moment, produced by the electric motor; $M_{\text {external }}$ - the moment of the external loads of the propeller relative to the rotation axis; $M_{\text {propeller }}$ - the propeller torque; $J_{P}$, $J_{M}$ - the moment of inertia of the rotor around the axis of the propeller and the axis of the motor, respectively; $\omega_{i}, \Omega_{i}$ - the angular velocity of the motor and propeller, respectively.

The differential equations for the four propellers that describe interrelated electromagnetic and mechanical processes in the electromechanical system of the convertiplane propeller drives are the following:

$$
\left\{\begin{array}{l}
J_{i} \frac{d \omega_{i}}{d t}=M_{\text {motor }}-M_{\text {external }} \\
L_{i} \frac{d i_{i}}{d t}+i_{i} R_{i}+c_{E} \omega_{i}=U_{i}
\end{array},\right.
$$

where $\mathrm{Li}, \mathrm{Ri}$ - the inductance and active resistance of the rotor winding; $i$ - the current in the winding of the electromagnet; $\mathrm{cE}$ - the coefficient of proportionality, called the constant EMF of the motor; $\mathrm{Ji}$ - the reduced axial moment of inertia; $\mathrm{Ui}$ - the control voltage.

The moment produced by the motors:

$$
M_{\text {motor }}=c_{M_{i}} \cdot i_{i}
$$

where $\mathrm{cMi}$ - the constant of the motor torque.

The connection between the angular velocity of the motor $\omega \mathrm{i}$ and the screw rotor propeller $\Omega \mathrm{i}$ :

$$
\omega_{i} / \Omega_{i}=N,
$$

where $\mathrm{N}$ - the contact ratio of the reduction.

The moment of the external loads $M_{\text {external }}$ is defined as:

$$
M_{\text {external }}=\frac{M_{\text {propeller }}}{N \cdot \eta}=\frac{\mu \Omega_{i}^{2}}{N \eta},
$$

where $\mathrm{MBi}$ - the moment of resistance to the propeller rotation, $\eta-$ the efficiency of the electric drive that connects the mechanical energy of the engine axis and the propeller, $\mu-$ the load factor, reduced to the motor shaft.

Solving approximately the system of equations (2), taking into consideration (3), (5) we get equations, relating the angular velocity of propeller rotors and the control voltage supplied to the motors [8]:

$$
\dot{\omega}_{i}=\frac{-\left(c_{E} c_{M} / R\right) \eta N^{2} \omega_{i}-d \omega_{i}^{2}+\left(c_{M} / R\right) \eta N U_{i}}{J_{p}+\eta N^{2} J_{M}}
$$

where $J_{P}, J_{M}$ - the moment of the rotor inertia around the axis of the propeller and the axis of the motor, respectively, $d$ - the aerodynamic constant.

\section{The synthesis of the energy efficient parameters of the electric drive}

As the criterion of the energy efficiency there will be chosen the following value:

$$
\varepsilon=\left|A_{C}\right| \frac{1}{\int_{0}^{T} W d t}
$$

where $\int_{0}^{T} W d t-$ the losses in electric drives, $T$ - the time of motion, $A_{c}-$ the work of the traction forces, the forces of gravity and resistance to movement at a given movement of the center of mass:

$$
A_{c}=\int_{0}^{T} \bar{R} \cdot \dot{\mathbf{r}}_{c} d t
$$

where $m$ - the total weight of the convertiplane, $g$ - the gravitational acceleration, $\mathbf{r}_{C}=\left[\begin{array}{llll}X_{C} & Y_{C} & Z_{C}\end{array}\right]^{T}-$ the vector, defining the position of the center of mass of the device, $\bar{R}=\bar{P}+\bar{Q}+\bar{G}-$ the main vector of external forces.

The value $W$ that determines the losses in electric drives in the formula (7), will be presented like:

$$
W=\left(\mathbf{u}^{*}-\mathbf{u}\right) \mathbf{R}\left(\mathbf{u}^{*}-\mathbf{u}\right)
$$

where $\mathbf{R}$ - the matrix of the given resistances of electric motors $\mathbf{u}=\left[\begin{array}{lll}M_{1} & M_{2} & M_{3}\end{array}\right]^{T}-$ the vector of control actions, and $\mathbf{u}^{*}=\left[\begin{array}{lll}M_{1}^{*} & M_{1}^{*} & M_{1}^{*}\end{array}\right]^{T}-$ the vector of control actions, which is necessary for obtaining the desired time dependences of the generalized coordinates $\mathbf{q}^{*}(t)$ : 


$$
\begin{gathered}
\mathbf{u}^{*}=\mathbf{B}^{-1}\left(\mathbf{H} \ddot{\mathbf{q}}^{*}+\mathbf{C}+\mathbf{G}\right), \\
\mathbf{u}=\mathbf{u}^{*}+\mathbf{B}^{-1} \mathbf{H}\left(\mathbf{K}_{p} \mathbf{e}+\mathbf{K}_{d} \dot{\mathbf{e}}\right),
\end{gathered}
$$

where $\mathbf{H}$ - the generalized matrix of inertia of the mechanism, $\mathbf{C}$ - the vector of the generalized Coriolis and normal inertial forces, $\mathbf{G}$ - the vector of the generalized gravity, $\mathbf{B}$ - the matrix that connects the vector of control actions with the vector of the generalized forces, generated by the drives, $\mathbf{K}_{p}$ и $\mathbf{K}_{d}-$ the diagonal matrix of coefficients of the regulator:

$$
\mathbf{K}_{p}=\left[\begin{array}{ccc}
k_{1} & 0 & 0 \\
0 & k_{2} & 0 \\
0 & 0 & k_{3}
\end{array}\right], \mathbf{K}_{d}=\left[\begin{array}{ccc}
k_{4} & 0 & 0 \\
0 & k_{5} & 0 \\
0 & 0 & k_{6}
\end{array}\right],
$$

where $k_{j}$ - the positive coefficients of the regulator $(j=\overline{1,6})$.

The values of the matrixes and vectors mentioned above, and the way of their finding are given in publications [12-15]. The general analysis methods of the equations of kinematics and dynamics of flying devices are shown in the following works [5-9]. The controller is described in the papers [7].

The currents in the windings of the electric drives are connected with the moments generated by the expression $C_{\tau} \eta I_{i}=M_{i}$, where $I_{i}$ - the current in the windings of the $i$-drive, $C_{\tau}-$ the constant of the electric motors moments, $\eta-$ the contact ratio of the reducer. The desired values of the currents $I_{i}^{*}$ will be determined according to the formula: $I_{i}^{*}=M_{i}^{*} /\left(\eta C_{\tau}\right)$, and $M_{i}^{*}$ will be received from the equation (10). The currents $I_{i}^{*}$ determine the energy loss $W^{*}=\sum_{i=1}^{3} I_{i}^{*} R_{i}$, with the motion of an exoskeleton on the desired trajectory, where $R_{i}$ - the resistance of windings of the $i$-drive. The value $W^{*}$ corresponds to the minimum energy loss at a given trajectory of the gravity center of the convertiplane. The value $W$ will be determined in a scalar form:

$$
W=\sum_{i=1}^{3}\left(I_{i}^{*}-I_{i}\right)^{2} R_{i} .
$$

Taking into account (13) the matrix of given resistances $\mathbf{R}$ is:

$$
\mathbf{R}=\left[\begin{array}{ccc}
R_{1} /\left(\eta^{2} C_{\tau}^{2}\right) & 0 & 0 \\
0 & R_{2} /\left(\eta^{2} C_{\tau}^{2}\right) & 0 \\
0 & 0 & R_{3} /\left(\eta^{2} C_{\tau}^{2}\right)
\end{array}\right]
$$

The formula (13) allows finding the additional losses in electric drives, resulting from the deviation of the real trajectory from the desired one. Minimizing $Q$ it is possible to improve the energy efficiency of a convertiplane. The control system of a convertiplane is described in [7, 8] and consists of the following blocks: The "Generator of master controls" that defines the desired trajectory of the gravity center of the convertiplane, the "Block of solution" that evaluates $\mathbf{q}^{*}(t)$, implementing this trajectory, the comparator that calculates the error of control $\mathbf{e}=\mathbf{q}^{*}-\mathbf{q}$, the controller and the control object - the model of the convertiplane.

The method of the flying vehicle control should provide greater efficiency under the condition of minimizing the deviation of the center of mass of the convertiplane from the desired trajectory of movement.

\section{The optimization of the control system parameters}

As the vector of variable parameters there will be chosen $\mathbf{k}=\left[\begin{array}{llllll}k_{1} & k_{2} & k_{3} & k_{4} & k_{5} & k_{6}\end{array}\right]^{T}$. The components of the vector $\mathrm{k}$ are defined on the intervals:

$$
0 \leq k_{j} \leq k_{\max , j}
$$

where $k_{\max , j}$ - the maximum allowable value of the controller coefficient, $j=\overline{1,6}$.

The target function $J=J(\mathbf{k})$ will be defined as a weighted sum of two integral performance criteria:

$$
\begin{gathered}
J=\mu_{1} J_{1}+\mu_{2} J_{2} \\
J_{1}=\int_{0}^{T}\left[\left(\mathbf{q}^{*}-\mathbf{q}\right)^{T}\left(\mathbf{q}^{*}-\mathbf{q}\right)\right] d t / \int_{0}^{T}\left[\left(\mathbf{q}^{*}\right)^{T}\left(\mathbf{q}^{*}\right)\right] d t, \\
J_{1}=\int_{0}^{T} W d t / \int_{0}^{T} W^{*} d t,
\end{gathered}
$$

where $\mu_{1}, \mu_{2}-$ the weight coefficients. By varying the coefficients, it is possible to obtain the regulators that can provide a greater precision, or greater energy efficiency. The criterion $J_{1}$ grows with the increase of the module of the control error e, and the criterion $J_{2}-$ with increasing of $\mathrm{W}$ value. Thus, minimizing $J$ provides the decrease of both the errors of control and the losses.

The task of determining the optimal parameters of the controller consists of finding the vector $\mathbf{k}_{m}$, leading $J$ to the minimum.

The methodology of determining the optimal parameters is focused on the method of sensing the space variable values which are determined by the values of criterion parameters at different points in space with a further solution of the problem of smooth surface's approximation of second order points.

\section{Conclusion}

As a result of the research, there has been proposed a model of a convertiplane, taking into account the optimal geometric parameters of the wings that meet the required aerodynamic characteristics and provide the increased maneuverability and the speed characteristics of this model. With the help of the XFLR5 software product 
there have been determined the coefficients of lift and resistance forces that will help determining the parameters of propellers and electric motors of the convertiplane.

There has also been formulated the task of the nonlinear optimization of the controller parameters of the convertiplane, implementing the flight in the vertical and horizontal planes. The objective function is defined as weighted sum of the two integral quality criteria related to accuracy and power consumption of the mechanical system. The optimization is accomplished by numerical methods of global optimization for different values of the weight coefficients of the objective function. It is shown that the optimal choice of the vector $\mathbf{k}_{m}$ reduces manifold the value of the objective function, increasing the accuracy of the automatic control system and reducing the power consumption.

\section{References}

1. G.S. Byushgens (Eds.). Flight Dynamics (Moscow, Mashinostroenie, 2011)

2. P.P. Afanasiev, I.S. Golubev et al. Unmanned aerial vehicles (Moscow, MAI, 2008)

3. N.F. Krasnov, Aerodynamics and wing and profile, In The basic theory of aerodynamics (Moscow, 1976)

4. M.L. Mil et al. Helicopters (Moscow, Mashinostroenie, 1967)

5. V.E Pavlovski, S.F. Jatsun, O.V. Emelyanova, S.P Stykanyova, Unmanned vehicle with elements of artificial intelligence (UV-II-2015), proceedings of the second all-Russian scientific-practical seminar, 99-106 (2015)

6. S.F. Jatsun, O.V. Emelyanov, A.I. Savin, S.P. Stykanyova, News SWSU, 14, 55 (2015)

7. S.F. Jatsun, O.V. Yemelyanov, K.G. Kazaryan, Unmanned vehicles with elements of artificial intelligence (UV-II-2016): proceedings of the third all-Russian scientific-practical seminar, 147-157 (2016)

8. V.E. Pavlovsky, S. F. Jatsun, O.V. Emelyanova, A.V. Savitsky, Robotics and technical cybernetics: scientific and technical journal, 5, 49-57 (2014)

9. S.F. Jatsun, O.V. Emelyanova, A.I. Savin, News of the SWSU. A series of engineering and technology, 17, 31-37 (2015)

10. The website "Podelise" http://tnu.podelise.ru/docs/ index $-385011 . h t m l$ ?page $=5$

11. The website "Airfoil Tools" http://airfoiltools.com/

12. The website "Soviet helicopters" http://wiki.nashtransport.ru/wiki/Советские_верто леты

13. G. Salazar-Cruz, Aerospace and Electronic Systems Conference (2008)

14. A. Oosedo, S. Abiko, S. Narasaki, A. Kuno et al. 2015 IEEE International Conference on Robotics and Automation (ICRA), 2326-2331 (2015)

15. S.F. Jatsun, S.V. Efimov et al. The patent for useful model No. 157967, IPC B64C 25/62. The air propulsion with variable thrust vectoring / (RF). No. 2015131037/11 (2015) 